\title{
Targeting of interleukin-13 receptor a 2 for treatment of head and neck squamous cell carcinoma induced by conditional deletion of TGF- $\beta$ and PTEN signaling
}

Bradford Hall', Hideyuki Nakashima², Zhi-Jun Sun', Yuki Sato², Yansong Bian ${ }^{1,3}$, Syed R Husain², Raj K Puri and Ashok B Kulkarni ${ }^{{ }^{*}}$

\begin{abstract}
Background: The sixth leading class of cancer worldwide is head and neck cancer, which typically arise within the squamous epithelium of the oral mucosa. Human head and neck squamous cell carcinoma (HNSCC) is known to be difficult to treat and has only a 50\% five-year survival rate. With HNSCC, novel therapeutics are needed along with a means of rapidly screening anti-cancer agents in vivo, such as mouse models.

Methods: In order to develop new animal models of cancer to test safety and efficacy of novel therapeutic agents for human HNSCC, tumors resembling clinical cases of human HNSCC were induced in the head and neck epithelium of a genetically engineered mouse model. This mouse model was generated by conditional deletion of two tumor suppressors, Transforming Growth Factor- $\beta$ Receptor 1 (TGF $\beta$ RI) and Phosphatase and Tensin homolog (PTEN), in the oral epithelium. We discovered that the tumors derived from these Tgfbr1/Pten double conditional knockout (2cKO) mice over-expressed IL-13Ra2, a high affinity receptor for IL-13 that can function as a tumor antigen. To demonstrate a proof-of-concept that targeted therapy against IL-13Ra2 expression would have any antitumor efficacy in this spontaneous tumor model, these mice were treated systemically with IL-13-PE, a recombinant immunotoxin consisting of IL-13 fused to the Pseudomonas exotoxin A.
\end{abstract}

Results: Tgfbr1/Pten 2CKO mice when treated with IL-13-PE displayed significantly increased survival when compared to the untreated control mice. The untreated mice exhibited weight loss, particularly with the rapid onset of tongue tumors, but the treated mice gained weight while on IL-13-PE therapy and showed no clinical signs of toxicity due to the immunotoxin. Expression of IL-13Ra2 in tumors was significantly decreased with IL-13-PE treatment as compared to the controls and the number of myeloid-derived suppressor cells (MDSC) was also significantly reduced in the spleens of the IL-13-PE treated mice.

Conclusions: Our study demonstrates that the Tgfbr1/Pten 2cKO mouse model of human HNSCC is a useful model for assessing antitumor activity of new cancer therapeutic agents, and that IL-13-PE has therapeutic potential to treat human head and neck cancer.

Keywords: IL-13 receptor, Immunotoxin, TGF- $\beta$, PTEN, HNSCC

\footnotetext{
* Correspondence: ak40m@nih.gov

${ }^{1}$ Functional Genomics Section, Laboratory of Cell and Developmental

Biology, National Institute of Dental and Craniofacial Research, National

Institutes of Health, 30 Convent Drive, Building 30, Room 130, Bethesda, MD,

USA

Full list of author information is available at the end of the article
} 


\section{Introduction}

In order to test new therapies for the prevention and treatment of head and neck squamous cell carcinoma (HNSCC), we generated a novel mouse model that mimics clinical cases of human head and neck cancer [1]. Our mouse model of HNSCC allows for conditional deletion of two important tumor suppressors in the oral epithelium: Transforming Growth Factor- $\beta$ Receptor 1 (TGF $\beta R I$ ), an inhibitor of epithelial proliferation, and phosphatase and tensin homolog (PTEN), an enzyme that negatively regulates PI3K/Akt signaling to prevent uncontrolled cell growth. Patients with human HNSCC often display alterations in the cellular signaling pathways associated with these two tumor suppressors [2-4]. Upon deletion of both TGFßRI and PTEN, the resulting double conditional knockout (2cKO) mice develop papillomas as early as 4 weeks after tumor induction, and these tumors progress to squamous cell carcinomas with $100 \%$ penetrance. The majority of the Tgfbr1/Pten 2cKO mice develop tumors on the epithelium of the tongue, although tumors also form elsewhere on the head and neck epithelium such as the ears and muzzle area. The tumors in the Tgfbr1/Pten 2cKO mice display many of the same biochemical alterations that are common to human HNSCC, particularly with regard to upregulation of inflammatory cytokines that promote tumor growth and proliferation.

Since HNSCC accounts for about $8 \%$ of newly diagnosed cancers worldwide [5] with an overall 5-year survival rate of only $40-50 \%$ in patients despite recent advances in multimodality therapy [6], novel therapeutic agents are needed that will increase overall survival while avoiding the toxicities associated with current radiotherapy and chemotherapy. We have previously reported that IL-13R $\alpha 2$, a receptor that binds IL-13 with high affinity, is uniquely overexpressed on some human head and neck cancers. The expression of the IL-13 receptors has been shown to be significantly higher in HNSCC than in normal tissues with a tissue array. In particular, about $33 \%$ of human HNSCCs display moderate to high-level expression of IL-13R $\alpha 2$ [7]. The expression of IL-13R $\alpha 2$, a unique receptor that binds IL-13 with high affinity and involved in signaling in cancer cells, is over expressed in cancer while its expression is mostly absent or low on other tissues except expression in testicular tissue [8]. We therefore, examined tumors from the Tgfbrl/Pten $2 \mathrm{cKO}$ mice for expression of IL-13R $\alpha 2$ in order to establish any further link between our mouse model and human cases of HNSCC. We discovered that this receptor is overexpressed within the squamous cell carcinomas of the mice. With this knowledge of IL-13R $\alpha 2$ upregulation in the tumors, we hypothesized that our mouse model could be used to study targeted therapy against this cancer marker.
One therapy that can be used to target expression of the IL-13R $\alpha 2$ tumor antigen involves the use of a novel recombinant immunotoxin, designated IL-13-PE, which consists of the IL-13 cytokine fused to the Pseudomonas exotoxin A (PE) [9]. IL-13-PE has been shown to have cytotoxic effects against head and neck cancer cell lines that are either positive for IL-13R $\alpha 2$ or are stably transfected to express IL-13R $\alpha 2$. Anti-tumor activity was demonstrated with IL-13-PE in an immunodeficient xenograft mouse model using these human HNSCC cell lines and the immunotoxin treatment were welltolerated by the mice [10]. With the sensitivity of these HNSCC cell lines to IL-13-PE, we decided to test this immunotoxin treatment in a genetically engineered mouse model of head and neck cancer, the Tgfbr1/Pten $2 \mathrm{CKO}$ mice. Because secretion of IL-13 can influence tumor suppressor cells including myeloid derived supressor cells leading to immunoevasion [11,12], testing IL-13-PE in the immunocompetent Tgfbr1/Pten 2cKO mice will allow us to study the effects of this treatment on the tumor microenvironment, particularly the interactions with the immune system. Also, there is a known interplay between IL-13 and TGF- $\beta$ signaling $[13,14]$, so it will additionally be interesting to examine IL-13-PE treatment in a context where, similar to a subset of human HNSCC patients, the cancer cells are unaffected by the growth inhibitory effects of TGF- $\beta 1$ and can thereby utilize this cytokine to create a tumorpromoting microenvironment. So, HNSCC was induced in the Tgfbr1/Pten 2cKO mouse model and mice were dosed with IL-13-PE to determine the therapeutic effect of this treatment. The Tgfbrl/Pten 2cKO mice that received IL-13-PE treatment had significantly increased longevity as compared to the controls. These results suggest that our spontaneous immunocompetent head and neck tumor model is useful for testing cancer therapeutics and that IL-13-PE could be a useful treatment regimen for inhibiting the growth of human HNSCC.

\section{Materials and methods Tgfbr1/Pten 2cKO mice}

The Tgfbr1/Pten 2cKO mice (K14-CreER ${ }^{\text {tam }}$ Tgfbrlf/f/ Ptenf $/ f$ ) were generated as previously described [1]. Essentially, tamoxifen $(200 \mu \mathrm{l}$ at a concentration of $10 \mu \mathrm{g} / \mu \mathrm{l}$ in corn oil) was applied to the oral cavity of 5- to 7-week-old K14-CreER ${ }^{\text {tam }}$; Tgfbrlf/f/Ptenf/f mice to induce the K14CreER ${ }^{\text {tam }}$ transgene which causes deletion of TGF $\beta R I$ and PTEN in the head and neck epithelium. Tumors developed in $100 \%$ of the mice as early as 4 weeks after conditional deletion of these tumor suppressors. When the lesions reached $2 \mathrm{~cm}$, or became ulcerated, the animals were euthanized and a necropsy was performed. Mice were also euthanized in the survival studies if animals exhibited any signs of excessive weight loss 
(cachexia), typically due to tongue tumors that obstruct proper eating. All care to the mice was given in compliance with the National Institutes of Health guidelines on the use of laboratory and experimental animals, and the studies were approved by the National Institute of Dental and Craniofacial Research (NIDCR) Animal Care and Use Committee (ACUC).

\section{IL-13-PE treatment}

A recombinant fusion IL-13 immunotoxin termed as IL13-PE, consisting of human IL-13 and a mutated form of Pseudomonas exotoxin, was purified in our laboratory as described previously [9]. Since human IL-13 binds to murine cells, this fusion protein was used in murine experiments [15]. IL-13-PE was administered to the Tgfbr1/Pten 2cKO mice by i.p. injection in a volume of $500 \mu \mathrm{l}(50 \mu \mathrm{g} / \mathrm{kg})$ in $0.2 \%$ human serum albumin in PBS [10]. The i.p. schedule included two injections per day (b.i.d.) at a minimum interval of 6-8 hr on alternate days for two weeks (a total of 12 injections per mouse).

\section{Cell culture and protein synthesis inhibition assay}

Primary cultures were established as previously described [16]. Excised tumors from the Tgfbr1/Pten 2cKO mice were enzymatically digested $(10 \mathrm{mg} / \mathrm{ml}$ collagenase, $1 \mathrm{mg} / \mathrm{ml}$ hyaluronidase, and $0.5 \mathrm{mg} / \mathrm{ml}$ DNAse [Sigma-Aldrich, St. Louis, MO]) and the isolated tumor cells were cultured in RPMI1640 medium containing 10\% FBS. After two to three passages, tumor cells were treated with IL13-PE and cytotoxicity was measured. Essentially, $10^{4}$ cells were cultured with or without various concentrations of IL-13-PE for 22 hours in a leucine-free medium and then incubated with $1 \mu \mathrm{Ci}$ of $\left[{ }^{3} \mathrm{H}\right]$ leucine (NEN Research Products, Waltham, MA) for an additional 4 hours. Following these incubation times, the uptake of the radioactive leucine was measured using a Beta plate counter (Wallace, Waltham, MA).

\section{Histology and immunohistochemistry}

Tumors along with control tissues such as buccal mucosa, tongue, spleen, lung, kidney, and liver were carefully dissected from the mice and fixed overnight in $10 \%$ buffered formalin. The tissues were embedded in paraffin and $5-\mu \mathrm{m}$ sections were used for histopathological analysis. Tumor pathology was examined using slides stained with hematoxylin and eosin (H\&E). For immunohistochemistry, sections were deparaffinized in xylene, rehydrated with descending grades of ethanol, blocked for $30 \mathrm{~min}$, and incubated with primary antibody (see below) prepared in Background Reducing Antibody Diluent (Dako, Carpentaria, CA). Next, the sections were treated with the Rabbit on Rodent HRP-Polymer (Biocare, Concord, CA) or with a biotinylated secondary antibody (Vector Laboratories, Burlingame, CA) followed by treatment with the
Vectastain ABC reagent (Vector Laboratories). Antibody complexes were detected using liquid DAB (Biogenex, Fremont, CA). Primary antibody dilutions are as follows: 1:100 dilution for anti-mouse IL-13R $\alpha 2$ (R\&D Systems, Inc., Minneapolis, MN), and 1:100 dilutions for phosphoAkt (Ser473) (D9E) antibody (Cell Signaling, Danvers, MA) and Anti-Mouse Ki-67 Antigen Clone TEC-3 (Dako, Carpentaria, CA).

\section{Western blot analysis}

Tumors and normal epithelium from the buccal mucosa and tongue were collected from Tgfbr1/Pten 2cKO and Tgfbrlf/f/Ptenf/f mice, and protein lysates were generated using T-PER reagent (Pierce, Rockford, IL) with a complete mini protease inhibitor cocktail (Roche, Branchburg, NJ). About $50 \mu \mathrm{g}$ of the extracted protein were denatured and then separated with electrophoresis on NuPAGE 4-12\% Bis-Tris precast gels using XCell surelock Mini-Cell (Invitrogen, Carlsbad, CA). Proteins were then transferred onto a nitrocellulose membrane, blocked for 1 hour, and incubated with primary antibodies overnight. The attached antibodies were then visualized using a horseradish peroxidase-conjugated secondary antibody (Santa Cruz, Santa Cruz, CA) followed by chemiluminescence detection (Pierce, Rockford, IL). Subsequently, all blots were re-incubated with anti-Actin antibody (1:5000 dilution; Millipore, Billerica, MA) as a loading control. The following primary antibody dilutions were used: 1:250 for muIL-13R $\alpha 2$ (R\&D Systems, Inc., Minneapolis, MN) and 1:1000 for phospho-Akt (Ser473) (D9E), and, phospho-Stat3 (Tyr705) (D3A7), antibodies (Cell Signaling, Danvers, MA).

\section{Quantitative real-time PCR}

Total RNA was extracted from the tongue tumors and adjacent control tongue tissue using 3 pairs of Tgfbrl/ Pten 2cKO mice as well as a Tgfbrlf/f/Ptenf/f control. RNA was isolated using the miRNeasy Mini kit (Qiagen, Valencia, CA). For mRNA analysis, $0.5 \mu \mathrm{g}$ of total RNA were reverse transcribed into cDNA and a tenth of this solution was then used per well with iQ SYBR Green Supermix (Bio-Rad, Hercules, CA) for real-time PCR (RT-PCR). Samples were run in quadruplicate using a Chromo4 PCR System (Bio-Rad). The IL-13 receptors (IL-13R $\alpha 2$, IL-13R $\alpha 1$, IL-4R $\alpha$ ) and a $\beta$-actin internal control were detected using QuantiTect Primers (Qiagen, Valencia, CA). RT-PCR to detect IL-13R $\alpha 2$ expression on the primary tumor cultures was performed as previously described [16].

\section{Flow cytometric analysis}

Splenocytes were isolated from three Tgfbr1/Pten 2cKO mice immediately following IL-13-PE treatment and then compared to the appropriate controls. Quantification of MDSCs (CD11b+ GR-1+) by FACS analysis was performed 
as previously described [17]. Essentially, 1 x $10^{6}$ splenocytes were evaluated using FITC-conjugated anti-CD11b and PerCP-Cy5.5-conjugated anti-Gr-1 Abs (e-Bioscience, San Diego, CA). All flow cytometric analyses were performed on a FACSCanto II (Becton Dickinson, San Jose, CA) flow cytometer.

\section{Statistical analysis}

The data was analyzed for statistical significance using Graph Pad Prism version 5.00 for Windows (Graph-Pad Software Inc, La Jolla, CA). Survival curves were generated using the Kaplan-Meier method and compared using the Log-rank (Mantel-Cox) Test. Two-way ANOVA was used to compare the weight change between IL-13-PE treated and untreated groups. A two-tailed Student's unpaired $t$ test was used to determine statistical significance for all other comparisons including the determination of significant differences in receptor expression by realtime PCR, IL-13R $\alpha 2$ expression by Western blot, and numbers of MDSCs using FACS.

\section{Results}

\section{Expression of IL-13Ra2 in Tgfbr1/Pten 2cKO mouse with} HNSCC

Deletion of TGF $\beta$ RI and PTEN in the oral epithelium of mice has been shown to lead to the formation of spontaneous tumors that resemble human HNSCC, including similar tumor promoting alterations in various cell signaling pathways [1]. As previously reported the tumors in these mice look like human HNSCC pathologically with atypical cells and differentiated keratin pearls. The mouse tumors also mirror the same molecular alterations found in human HNSCC such as EGFR overexpression and activated Akt, NF-kB, and Stat3 signaling. Since IL-13R $\alpha 2$ is over-expressed in about $33 \%$ of human HNSCCs [7], we decided to examine the tumors of these mice for this unique cancer marker. Western blot showed that IL-13R $\alpha 2$ is upregulated in the tumors of the Tgfbr1/Pten 2cKO mice (Figure 1A). Immunohistochemistry analysis also showed staining of SCC from tongue and muzzle tumors but not in the normal tongue (Figure 1B). As previously described, HNSCC in the Tgfbr1/Pten 2cKO mice can be identified with activated Akt due to PTEN deletion, along with phosphorylated Stat3, both cell signaling pathways that are commonly activated in human HNSCC [1]. These two markers were used to verify that the IL-13R $\alpha 2$ expression is primarily restricted to the HNSCC. The tumors that arise because of TGF $\beta R I$ and PTEN deletion occur not only on the tongues of the mice, but also on the head and neck epithelium around the ears and the muzzle. Regardless of location, all squamous cell carcinomas displayed high expression of IL-13R $\alpha 2$, making the mouse model ideal for studying targeted cytotoxin therapy against IL-13R $\alpha 2$. Using real-time PCR, we also examined three sets of mice for the expression of transcripts for various chains of receptors, e.g. IL-13R $\alpha 2$, IL-13R $\alpha 1$, and IL-4R $\alpha$, which are involved in IL-13 receptor structure and function. We observed an upregulation of the transcripts of all three subunit receptors in the tongue tumors, as compared to the normal corresponding adjacent tongue tissue (Figure 1C). Expression of the IL-13 receptors was significantly higher in the tongue tumors from the Tgfbr1/Pten 2cKO mice ( $\mathrm{P}$ value $<0.0001$ ) when compared with the normal tongue tissue in which TGFBR1 and PTEN was not genetically deleted.

\section{Cytotoxicity of IL-13-PE on primary tumor cells from Tgfbr1/Pten 2cKO mice}

Since IL-13R $\alpha 2$ binds to IL-13 with high affinity and then undergoes internalization, expression of this receptor can thereby be targeted for delivery of a bacterial toxin into a cancer cell [18]. The therapeutic agent IL13-PE was designed to bind to cells expressing IL-13 receptors and cause selective cytotoxicitiy upon internalization. Primary cultures were derived from the tumors of the Tgfbr1/Pten 2cKO mice to determine if the upregulated expression of IL-13R $\alpha 2$ by the cancer cells would make them sensitive to the IL-13-PE cytotoxicity. The isolated primary cancer cells from the Tgfbr1/Pten $2 \mathrm{cKO}$ mice retain higher expression of IL-13R $\alpha 2$ as compared to other tissues, including the spleen, lung, kidney, liver, and skin (Figure 2A). Higher expression of IL-13R $\alpha 2$ is therefore primarily limited to the tumor cells and does not appear to be aberrantly triggered in any other tissues through the tumor induction process, which causes conditional deletion of TGF $\beta R I$ and PTEN. As seen in Figure 2B, the cultured primary tumor cells with upregulated IL-13R $\alpha 2$ were sensitive to the addition of IL-13-PE in a dose-dependent manner. Using two Tgfbr1/Pten 2cKO mice, each with multiple tumor sites, an $\mathrm{IC}_{50}$ (the concentration of drug causing $50 \%$ inhibition of protein synthesis) ranging between 45-100 ng/ml was determined for IL-13-PE. For this in vitro testing, PMRCC was selected as a positive control out of many cell lines tested because of higher expression of high-affinity IL-13R $\alpha 2$. Based on sensitivity $\left(\mathrm{IC}_{50}\right)$ of primary cells to IL-13-PE, these cells most likely express lower number of IL-13 receptors. We have not tested affinity of IL-13-PE binding on these cells. However, in previous studies, we demonstrated that higher binding affinity of IL-13-PE to tumor cells did not enhance cytotoxicity to cells because internalization of only few molecules of IL-13-PE was enough to kill the cells [19]. The primary tumor cultures seem to have an intermediate expression of IL-13R $\alpha 2$ where the $\mathrm{IC}_{50}$ for these cells is similar to the murine sarcoma cell line MCA304, which has moderate sensitivity to 


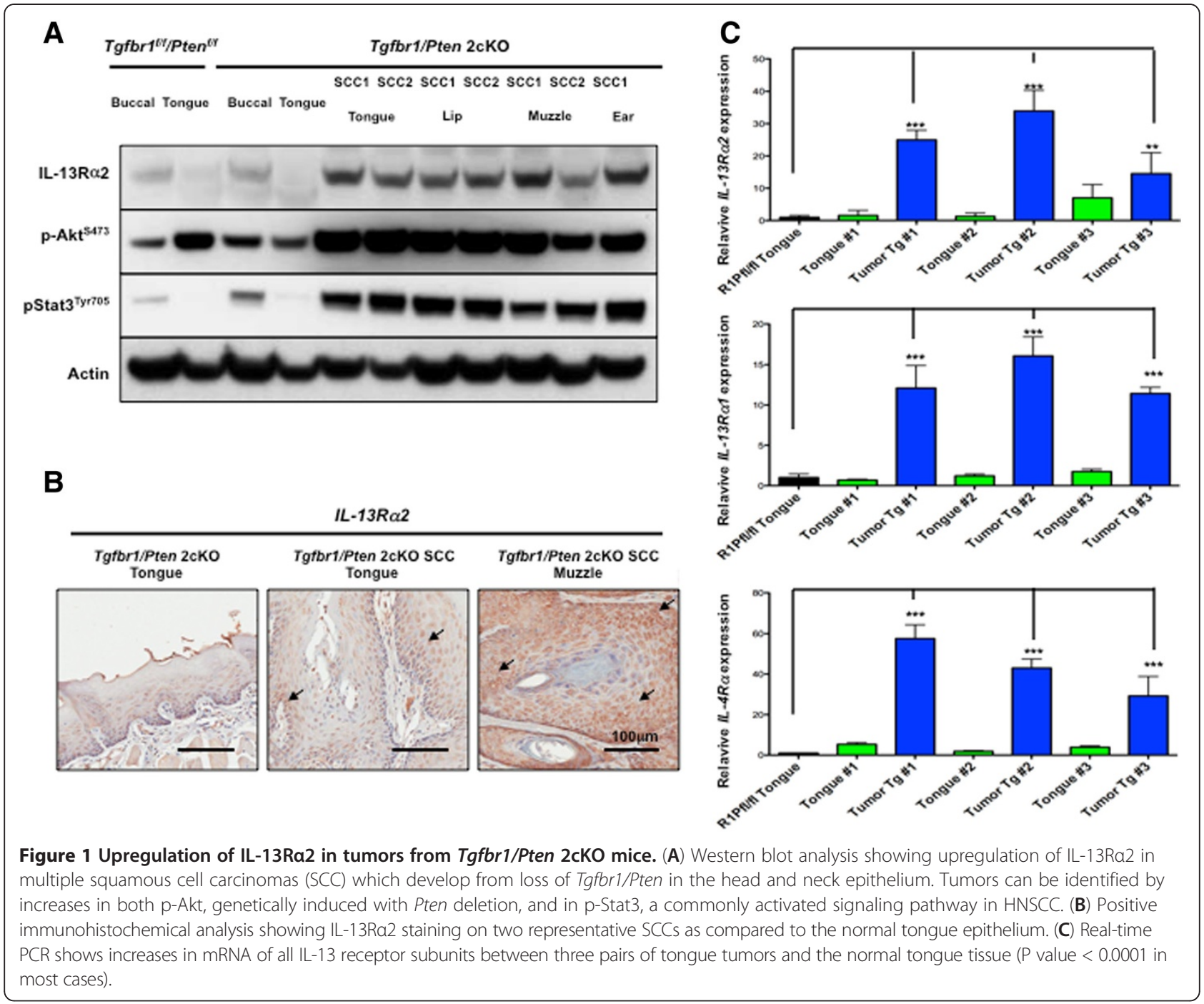

IL-13-PE [20]. Human gingival fibroblasts (HGF), which don't display IL-13R $\alpha 2$, were unaffected by IL-13-PE treatment. It is to be noted that cytotoxicity of IL-13-PE toward IL-13R $\alpha 2$ positive cells is highly specific as we have shown in our previous several studies that an excess of IL-13 (100-200 fold) neutralizes activity of IL-13-PE [10,21].

\section{IL-13-PE treatment of Tgfbr1/Pten 2cKO mice}

With confirmation of the cytotoxic effects of IL-13-PE on cultured primary murine HNSCC tumor cells, we next decided to treat Tgfbr1/Pten 2cKO mice with i.p. injections of the recombinant immunotoxin. The dosing schedule of IL-13-PE administration is illustrated in Figure 3A. Essentially, tumors were induced in mice between 5 to 7 weeks of age using daily oral administration of Tamoxifen for five days to promote the recombinase activity of the K14-CreER ${ }^{\text {tam }}$ transgene. The K14-CreER $R^{\text {tam }}$ in turn causes recombination in the Tgfbrlfff and Ptenf/f alleles and disrupts the expression of these two important tumor suppressors. Treatment with IL-13-PE was then initiated four weeks from the day tumor induction began, since papillomas and early carcinomas generally start to appear at this time in the Tgfbr1/Pten 2cKO mouse. The Tgfbr1/Pten 2cKO mice were dosed with two i.p. injections of IL-13-PE $(50 \mu \mathrm{g} / \mathrm{kg})$ per day (b.i.d.) at a minimum interval of 6-8 $\mathrm{hr}$ on alternate days for two weeks. The dose selected for this study was less than the maximum tolerated dose for IL-13-PE based on several previous mouse experiments [10]. The preclinical safety studies characterizing the organ toxicities stemming from higher doses of IL-13-PE have already been described previously [22,23]. The Tgfbr1/Pten 2cKO mice treated with IL-13-PE $(\mathrm{n}=25)$ showed increased survival $(\mathrm{p}=0.002)$ compared to the littermate controls that were injected only with excipient $(0.2 \%$ human serum albumin in PBS) $(n=23)$ (Figure 3B). IL-13-PE was administered to the mice as they first started to succumb to tumor-associated 

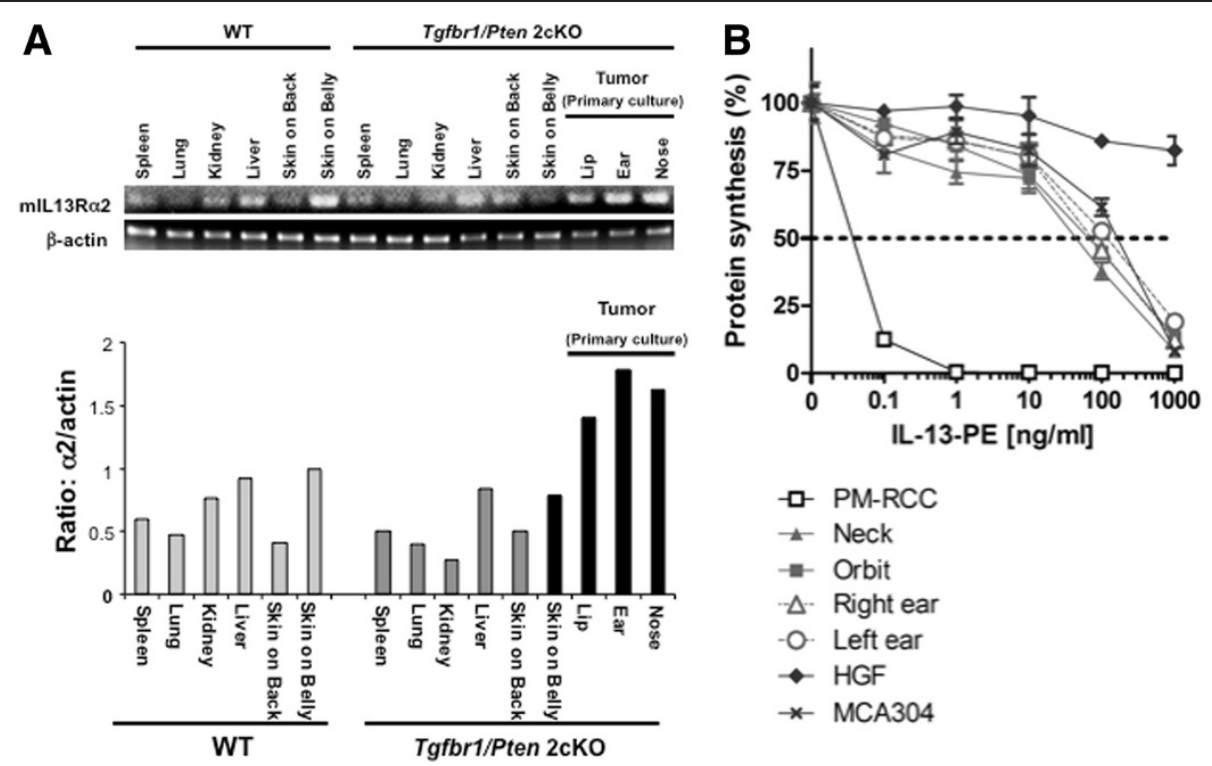

Figure 2 Sensitivity of primary tumor cultures to IL-13-PE treatment. (A) RT-PCR shows that IL-13Ra2 expression is high for the primary cultures but low in other tissues from Tgfbr1/Pten 2cKO mice undergoing tumor induction. (B) Primary cultures are sensitive to the cytotoxic effects of IL-13-PE, as measured by a decrease in protein synthesis. An IC 50 for IL-13-PE ranged between 45-100 ng/ml in the primary cultured tumor cells overexpressing IL-13Ra2.

mortality from the developing squamous cell carcinomas. The median survival significantly increased from 69 days after tumor induction in the controls to about 92 days through IL-13-PE treatment. At day 90, around the day of the median survival, 14 out of 25 (or $56 \%$ ) survived in the IL-13-PE treatment group compared to 5 out of 23 (or 21\%) in control group. More than twice as many treated mice were surviving at this time point as compared to the controls, clearly indicating the delay in progression of disease. In these survival studies, about $65 \%$ of the mice develop tongue tumors that impair the ability to eat and therefore cause cachexia. The mice given the IL-13-PE therapy had roughly the same occurrence of tongue tumors as the controls, so the immunotoxin treatment most likely delayed the development of these tumors, which accounts for the increased survival. As previously reported, metastasis was not observed in the Tgfbr1/Pten 2cKO cancer model because of the rapid growth and progression of the carcinomas that develop in these mice, so the effects of IL-13-PE treatment on metastasis could not be observed [1].

The IL-13-PE administration was well-tolerated by the Tgfbr1/Pten 2cKO mice and the mice treated with cytotoxin therapy showed improvements in their health, as opposed to the control littermates. In the few mice that had tumors at the onset of IL-13-PE treatment, the tumors appeared to regress after immunotoxin administration, in contrast to the untreated mice. Mice treated with IL-13-PE continued to gain weight while the controls lost weight due to the developing head and neck tumors ( $\mathrm{P}$ value $<0.0001$; Figure $3 \mathrm{C}$ ). In particular, mice with carcinomas on the tongue lost weigh due to an inability to eat properly. No histological toxicity was detected in association with IL-13-PE treatment in tissues such as the spleen, lung, and heart (data not shown), which thereby demonstrated the restricted targeting of the IL-13-PE cytotoxin to only the cancer cells and not the normal tissues. The receptor-targeted specificity of IL-13-PE has been shown earlier by using an irrelevant IL2-PE immunotoxin as a control. IL2-PE, a immunotoxin known to exert its cytotoxicity through binding to IL-2 receptor $\gamma$ chain was not cytotoxic to IL-13R $\alpha 2$ positive tumor cells confirming that IL-13 -PE-mediated cytotoxicity is IL-13R $\alpha 2$ specific [24].

\section{Decreased expression of IL13Ra2 after IL-13-PE treatment} Immunotoxin therapy by IL-13-PE will essentially target cancer cells that upregulate IL-13R $\alpha 2$ and will therefore select against its expression. We looked at the tumor tissues from treated Tgfbr1/Pten 2cKO mice to see if the expression of IL-13R $\alpha 2$ was decreased. Expression of IL-13R $\alpha 2$ was variable in the tumors collected from the Tgfbr1/Pten 2cKO mice but was generally decreased overall in the IL-13-PE treated mice (Figure 4A). In the IL-13-PE treated mice, the levels of IL-13R $\alpha 2$ were significantly decreased in tumors that developed on the head and neck epithelia such as the muzzle and ears ( $\mathrm{P}$ value $<0.05$; Figure $4 \mathrm{~B}$ ), but no significant difference 


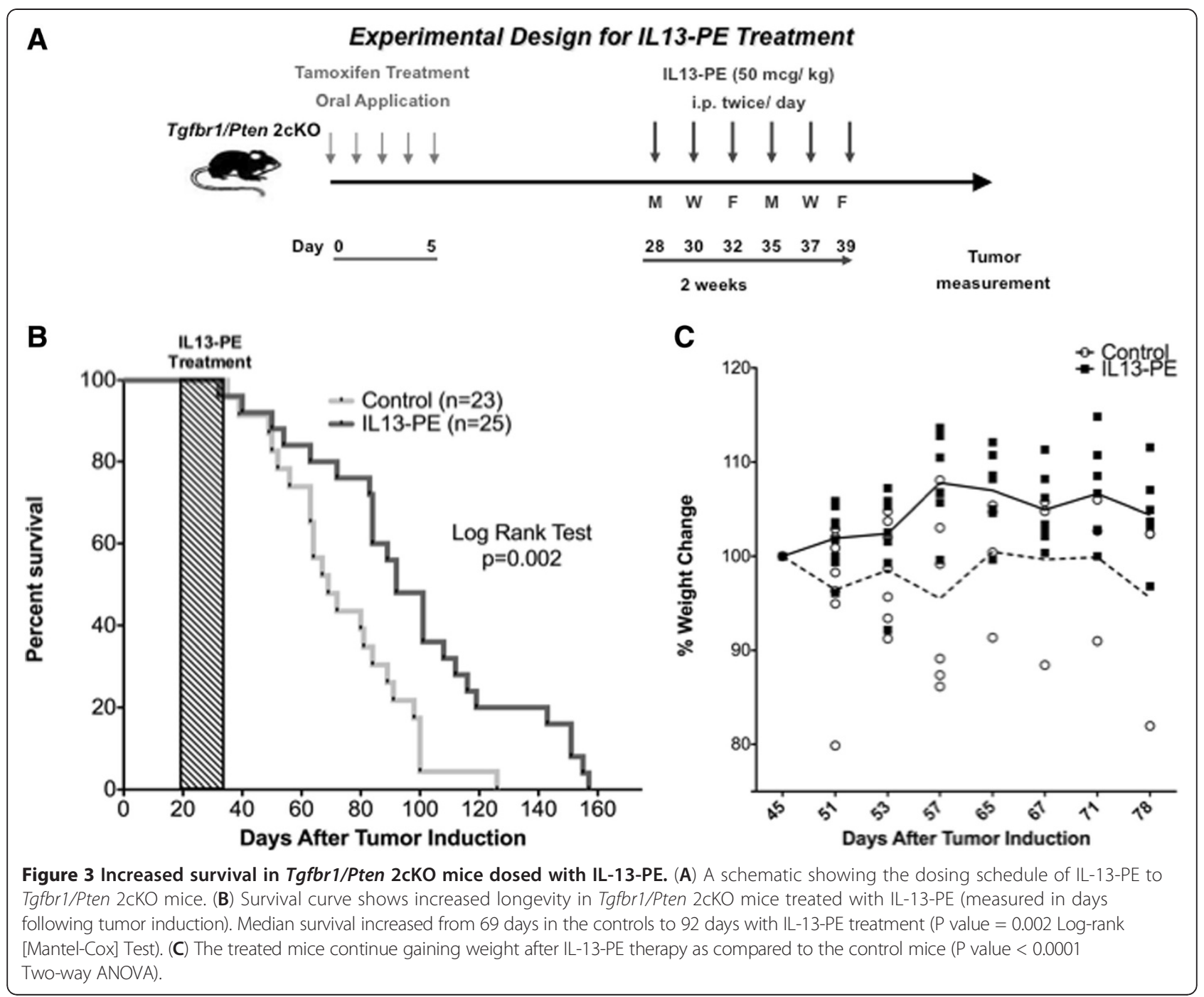

was detected for squamous cell carcinomas on the tongue (data not shown). Decreased expression of IL-13R $\alpha 2$ in the tumors was also detected through immunohistochemical analysis (Figure 4C) but, of the cancer cells that evaded cell death by IL-13-PE, no difference in proliferation could be seen with Ki67 staining as compared to the untreated mice. In addition, IL-13-PE had no significant effect on phosphorylation of Akt, a tumor promoting kinase that is irreversibly activated in the Tgfbr1/Pten 2cKO mice due to genetically Cre-mediated PTEN deletion. No association could be made between the level of IL-13R $\alpha 2$ on a tumor and the time since administering IL-13-PE treatment. Treatment with IL-13-PE at an early time point in cancer development may have selectively hindered the growth and establishment of high IL-13R $\alpha 2$ expressing cancer cells in the mice, resulting in the formation of tumors with decreased overall expression of this receptor.

\section{Reduction in MDSCs but not Tregs through IL-13-PE} treatment

The expression of IL-13R $\alpha 2$ has been linked to deleterious immune effect such as tumor immune evasion, particularly since signaling through this receptor causes upregulation of the immunosuppressive cytokine TGF$\beta 1[11,12]$. To examine for any immunological changes in the treated mice, some animals were sacrificed three days after the last dose of IL-13-PE, and FACS analysis was performed using the splenocytes. The MDSCs, a heterogeneous population of myeloid cells known to promote tumor immune evasion, were detected with FACS analysis using the monocyte/macrophage markers $\mathrm{CD} 11 \mathrm{~b}+$ and the granulocyte antigen $\mathrm{Gr}-1+$ [17]. The Tgfbrlf/f/Ptenf/f mice lacking the K14-CreER ${ }^{\text {tam }}$ transgene to cause conditional deletion were also analyzed as normal controls without tumors. As expected, the numbers of MDSCs in the spleens increased from 


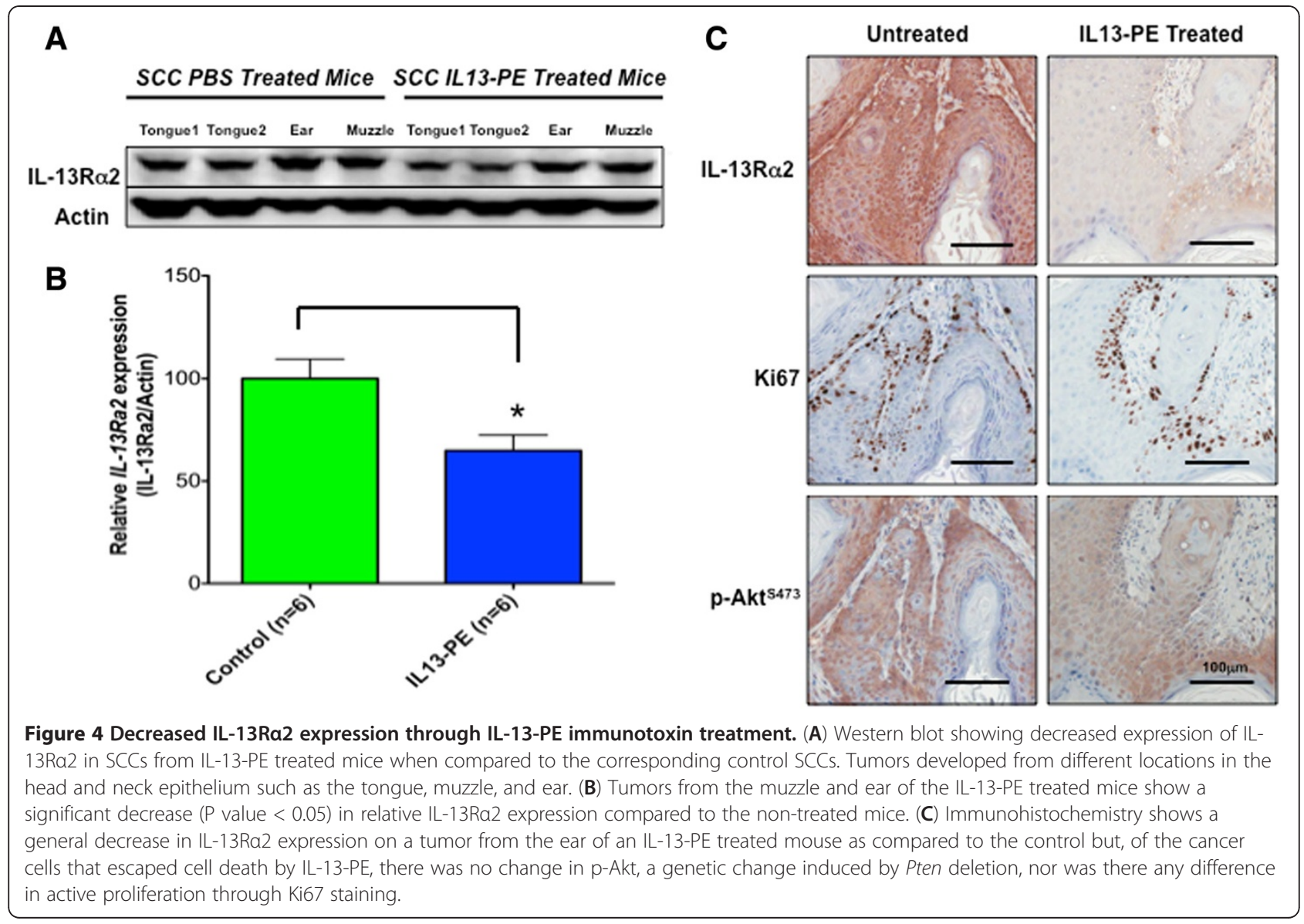

about $4.3 \%$ in the Tgfbr $1 f / f / P$ tenf/f mice to $32 \%$ in the untreated Tgfbr1/Pten 2cKO mice due to deletion of TGFßRI and PTEN and to the subsequent tumor development. Interestingly, the number of MDSCs dropped by half to $15.5 \%(\mathrm{P}<0.005)$ with IL-13-PE treatment (Figure 5B). A representative FACS plot is shown in Figure 5A.

Although the number of MDSCs decreased with IL13-PE treatment, no significant change was seen in the number of macrophages from the spleens of the mice (data not shown). Another immunosuppressive population of cells, $\mathrm{T}$ regulatory cells (Tregs), also showed no change between the two groups of mice (data not shown). The MDSCs, however, may be particularly sensitive to IL-13-PE treatment since IL-13R $\alpha 2$ has been shown to express on some populations of these myeloid cells in order to induce TGF- $\beta 1$ secretion and promote tumor immune evasion [11].

\section{Discussion}

In order to aid in the discovery of new treatments and mechanisms of human HNSCC actions, we have developed a mouse model that spontaneously forms tumors in the head and neck epithelium with 100\% penetrance. We observed that these tumors mimic human HNSCC with similar morphology and altered cell signaling. With such resemblance to human HNSCCs, the Tgfbr1/Pten 2cKO mice could be useful for screening novel therapeutics against cancer. Cancer cells often overexpress cell surface receptors of both growth factors and cytokines that help promote tumor growth, proliferation, and immune evasion. As previously shown, IL-13R $\alpha 2$ is upregulated in about 33\% of human HNSCC [7] but remains absent or shows very low expression on normal cells. Our previous in vitro studies showed that IL-13R $\alpha 2$ positive HNSCC cell lines were sensitive to cytotoxic effect of IL-13-PE depending upon the level of their expression of IL-13R $\alpha 2$ chain. The specificity and requirement of IL-13R $\alpha 2$ chain for cytotoxicity was further confirmed by transient transfection of IL-13R $\alpha 2$ chain in two IL$13 R \alpha 2$-negative HNSCC cell lines. Both transfectants acquired sensitivity to IL-13-PE confirming the relevance of the utility of IL-13-PE in head and neck cancer therapy [25]. We demonstrate that tumors from Tgfbr1/Pten 2cKO mice express high levels of not only IL-13R $\alpha 2$, but of IL-13R $\alpha 1$ and IL-4R $\alpha$ as well. We have previously shown that cultured cells derived from the periorbital and perianal squamous cell 

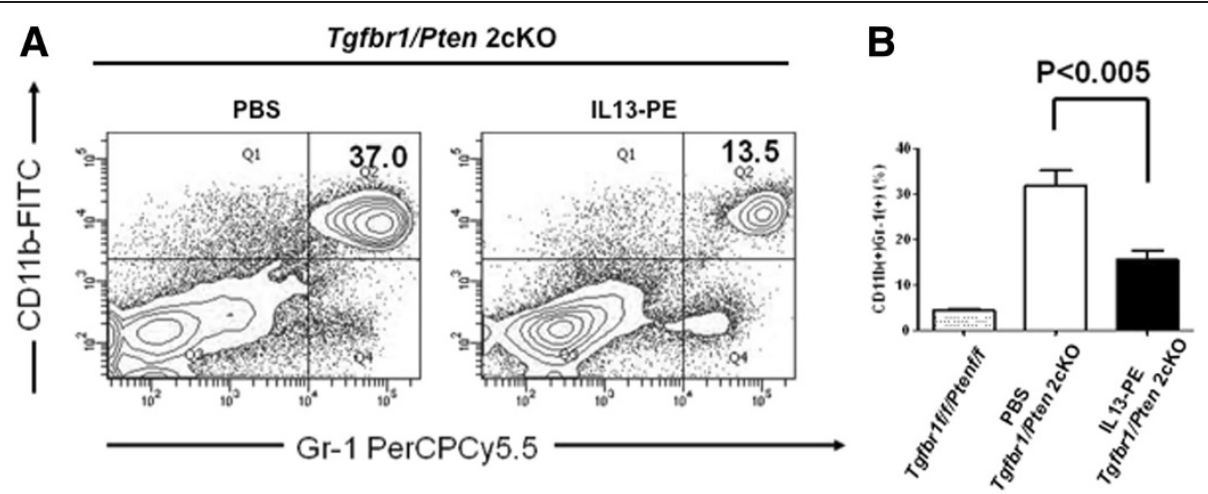

Figure 5 MDSCs were decreased by IL-13-PE treatment in Tgfbr1/Pten 2cKO mice. (A) Representative flow profile showing decreased populations of CD11b+Gr-1+ cells through IL-13-PE treatment. (B) Quantification of flow cytometry results. IL-13-PE treated mice are compared with both the saline injected Tgfbr1/Pten 2cKO tumor-bearing controls and the Tgfbrif/f/Ptenf/f mice without tumors. MDSCs were significantly reduced with IL-13-PE treatment ( $P$ value $<0.005)$.

carcinomas collected from another TGFßRI conditional knockout model can also express IL-13R $\alpha 2$ and are sensitive to IL-13-PE treatment [16]. Future studies will be conducted to determine if TGF $\beta$ RI deletion facilitates IL-13R $\alpha 2$ expression on squamous cell carcinomas. Because cell surface receptors can be targeted with monoclonal antibodies and fusion toxins, we tested whether the expression of the IL-13 receptors in tumors derived from Tgfbr1/Pten 2cKO mice can be targeted as part of a strategy to treat HNSCC. We found that a recombinant IL-13-PE immunotoxin targeting IL-13R $\alpha 2$ has remarkable anti-cancer activity against these tumors.

IL-13-PE immunotoxin is specifically designed to target tumors expressing this receptor by combining the IL-13 cytokine with the Pseudomonas exotoxin [9]. Bacterial toxins are ideal for generating immunotoxins since they can be easily produced in E. coli and show few side effects in humans if properly targeted [26]. Upon endocytosis, the fusion toxin translocates to the cytosol and inactivates elongation factor 2 to cause cell death. IL$13 R \alpha 2$ is also an ideal target for cytotoxin-based therapy since it is a unique tumor target displaying a high affinity for IL-13 and undergoes endocytosis upon binding. These qualities minimize the uptake of IL-13-PE by normal cells. Targeting IL-13R $\alpha 2$ expression may also be useful in restoring proper immunosurveillance [11].

As an anti-cancer strategy, IL-13-PE immunotoxin treatment has already proven to be efficacious against tumors in many cancer mouse models and is currently being used in clinical trials. IL-13-PE was used in a Phase 3 clinical study to treat patients with glioblastoma multiforme, another cancer type wherein IL-13R $\alpha 2$ is overexpressed in the majority of tumor cells. Convection-enhanced delivery of IL-13-PE was well-tolerated by patients with malignant gliomas [27]. One challenge of immunotoxin and any targeted therapy is efficient penetration into solid tumors.
Nevertheless, in the Tgfbr1/Pten 2cKO mouse model of HNSCC, systemic administration of IL-13-PE resulted in significantly increased survival in the treated mice as compared to the untreated controls. Tumor regression was evident in the few mice that had visible tumors at the start of IL-13-PE, particularly in the SCCs around the muzzle. Unlike the controls, the mice treated with the IL-13-PE group did not experience the immediate weight loss that typically occurs during tongue tumor development due to obstructed eating. However, both groups of mice in the survival studies eventually had the same occurrence of SCCs on the tongue, suggesting a possible delay in the formation and growth of these tumors. The weight gain together with the lack of any histological signs of toxicity also demonstrate that the IL-13-PE treatment is well-tolerated by the mice. In our recent study on the role of IL-13R $\alpha 2$ in pancreatic cancer invasion and metastasis, we demonstrated that IL$13 R \alpha 2-$ positive cancer metastasized to lymph nodes, liver, and peritoneum at a significantly higher rate compared with IL-13R $\alpha 2$-negative tumors. The level of IL-13R $\alpha 2$ expression in metastatic lesion was higher compared to the primary tumors [28]. Though we did not investigate the presence of metastasis in this model, the pancreatic tumor cells collected from lymph nodes metastasis were much more sensitive to IL-13-PE in vitro than compared with primary tumor cells indicating higher level of IL-13R $\alpha 2$ in metastatic lesion. Based on this data, we can predict similar efficacy of IL-13-PE to eliminate metastatic lesions common in patients with HNSCC. Future studies will be conducted to determine the optimal schedule and dose of administration of IL-13-PE.

It is interesting to note that IL-13R $\alpha 2$ expression was decreased in the tumors of IL-13-PE treated mice, indicating that high IL-13R $\alpha 2$ positive cells are eliminated while IL-13R $\alpha 2$ negative or low expressing tumors were 
not. However, this selective depletion of tumor cells did not cause any impact on proliferation of tumor cells as no difference in Ki67 staining was observed in tumors of treated mice compared to those of the untreated mice.

To examine the mechanism of antitumor response by IL-13-PE, we investigated whether treatment affected immunosurveillance to subsequently allow for enhanced tumor destruction by the immune system. IL-13-PE treatment caused a significant decrease in MDSCs in the spleen of treated mice that corresponded with increased survival. On the other hand, another immunosuppressive population of cells, Tregs, showed no change in the spleens between the two groups of mice (data not shown). This may be due to a direct cytotoxic effect of IL-13-PE on MDSCs, since some of these myeloid cells are known to express IL-13R $\alpha 2$ in order to induce TGF$\beta 1$ secretion and promote tumor immune evasion [12]. Future studies will examine the status of MDSCs and T regs in various tumors of these mice. However, it is possible that the reduction in the number of MDSCs resulted in improved immunosurveillance and, consequently, enhanced survival of the animals.

Taken together, the results from the Tgfbrl/Pten 2cKO mice validate our previous report on nude mice with transplanted tumors, which suggests that IL-13-PE may be an effective therapeutic agent for the treatment of HNSCC. Along with delaying tumor formation, we were able to see in the immunocompetent Tgfbr1/Pten 2cKO mice that the IL-13-PE treatment could additionally limit the development of an immunosuppressive tumor environment. Other treatment delivery options for IL-13-PE could improve the effectiveness of this immunotoxin in the Tgfbr1/Pten 2cKO mice such as direct injection into the head and neck tumors or a surgically implanted continuous infusion pump [22]. IL-13 -PE has also been shown to work synergistically with paclitaxel in an immunodeficient animal model of HNSCC [29] with gemcitabine for pancreatic cancer [30] and a DNA vaccine of IL-13R $\alpha 2$ in melanoma, breast, and sarcoma tumor models [20]. It is therefore possible that any of these combinations would be useful for testing the Tgfbr1/Pten 2cKO mouse model of spontaneous cancer. Further studies will be performed to test this combination approach. In summary, our study demonstrates that our HNSCC mouse model is valuable for developing novel cancer therapeutic approaches [31], and that IL-13-PE has therapeutic potential to treat human head and neck cancer.

\section{Conclusion}

Conditional deletion of both TGF $\beta$ RI and PTEN in the oral cavity of mice results in the formation of spontaneous squamous cell carcinomas in the head and neck area that display IL-13R $\alpha 2$, a tumor antigen expressed in $33 \%$ of human HNSCCs. Primary cultures from the Tgfbr1/Pten $2 \mathrm{cKO}$ mouse tumors show sensitivity to IL-13-PE. The Tgfbr1/Pten 2cKO mice were therefore tested with IL13-PE (50 $\mu \mathrm{g} / \mathrm{kg}$ b.i.d. on alternate days) for two weeks starting at an early point in tumor induction when carcinomas are first beginning to appear. This IL-13-PE regimen significantly increased survival in the tumor bearing mice ( $\mathrm{P}$ value 0.002 ) with no signs of toxicity. Expression of IL-13R $\alpha 2$ was reduced in the tumors while, concurrently, the spleens of the treated mice show reduced numbers of MDSCs, a population of myeloid cells that aid in tumor immune evasion. The increased survival of the IL-13-PE treated mice helps to further validate the idea that targeted therapy against IL-13R $\alpha 2$ expression could be employed as a clinical means of inhibiting HNSCC.

\section{Competing interests}

The authors declare that they have no competing interests.

\section{Authors' contributions}

Conception and design: BH, HN, Z-JS, SRH, RKP, ABK. Development of methodology: $\mathrm{BH}, \mathrm{HN}, \mathrm{SRH}, \mathrm{YB}, \mathrm{Acquisition}$ of data (provided animals, acquired and managed patients, provided facilities, etc.): BH, HN, Z-JS, YS, ABK, Analysis and interpretation of data (e.g., statistical analysis, biostatistics, computational analysis): BH, HN, SRH, RKP, ABK, Writing, review, and/or revision of the manuscript: B. Hall, S.R. Husain, R.K. Puri, A.B. Kulkarni, Administrative, technical, or material support (i.e., reporting or organizing data, constructing databases): SRH, YB, Study supervision: RKP, ABK. All authors read and approved the final manuscript.

\section{Acknowledgements}

We thank Riddhi Patel for help with the biochemical analysis of tumors, Alfredo Molinolo for the analysis of the tumor sections, and Shelagh Johnson for expert editorial assistance. We are grateful to Drs. Jing Han and ShyhChing Lo, CBER, FDA for internal review of the manuscript. These studies were supported by the Division of Intramural Research of the National Institute of Dental and Craniofacial Research, NIH to ABK (ZIA-DE-000698), and the Center for Biologics Evaluation and Research, U.S. Food and Drug Administration.

\section{Grant support}

Intramural Research Programs of the National Institute of Dental and Craniofacial Research and National Institute on Deafness and Other Communication Disorders, NIH, and the Center for Biologics Evaluation and Research, U.S. Food and Drug Administration.

\section{Author details}

${ }^{1}$ Functional Genomics Section, Laboratory of Cell and Developmental Biology, National Institute of Dental and Craniofacial Research, National Institutes of Health, 30 Convent Drive, Building 30, Room 130, Bethesda, MD, USA. ${ }^{2}$ Tumor Vaccines and Biotechnology Branch, Division of Cellular and Gene Therapies, Center for Biologics Evaluation and Research, U.S. Food and Drug Administration, Bethesda, MD, USA. ${ }^{3}$ Tumor Biology Section, Head and Neck Surgery Branch, National Institute on Deafness and Other

Communication Disorders, National Institutes of Health, Bethesda, MD, USA.

Received: 27 December 2012 Accepted: 11 February 2013

Published: 19 February 2013

\section{References}

1. Bian Y, Hall B, Sun ZJ, Molinolo A, Chen W, Gutkind JS, Waes CV, Kulkarni AB: Loss of TGF- $\beta$ signaling and PTEN promotes head and neck squamous cell carcinoma through cellular senescence evasion and cancer-related inflammation. Oncogene 2011, 31:3322-32. 
2. Molinolo AA, Amornphimoltham P, Squarize $\mathrm{CH}$, Castilho RM, Patel V, Gutkind JS: Dysregulated molecular networks in head and neck carcinogenesis. Oral Oncol 2009, 45:324-34.

3. Eisma RJ, Spiro JD, von Biberstein SE, Lindquist R, Kreutzer DL: Decreased expression of transforming growth factor beta receptors on head and neck squamous cell carcinoma tumor cells. Am J Surg 1996, 172:641-5.

4. Fukai Y, Fukuchi M, Masuda N, Osawa H, Kato H, Nakajima T, Kuwano H: Reduced expression of transforming growth factor-beta receptors is an unfavorable prognostic factor in human esophageal squamous cell carcinoma. Int J Cancer 2003, 104:161-6.

5. Jemal A, Bray F, Center MM, Ferlay J, Ward E, Forman D: Global cancer statistics. CA Cancer J Clin 2011, 61:69-90.

6. Bauman JE, Michel LS, Chung CH: New promising molecular targets in head and neck squamous cell carcinoma. Curr Opin Oncol 2012, 24:235-42.

7. Kawakami M, Kawakami K, Kasperbauer JL, Hinkley LL, Tsukuda M, Strome SE, Puri RK: Interleukin-13 receptor alpha2 chain in human head and neck cancer serves as a unique diagnostic marker. Clin Cancer Res 2003, 9:6381-8.

8. Debinski W, Gibo DM: Molecular expression analysis of restrictive receptor for interleukin 13, a brain tumor-associated cancer/testis antigen. Mol Med 2000, 6:440-9.

9. Joshi BH, Puri RK: Optimization of expression and purification of two biologically active chimeric fusion proteins that consist of human interleukin-13 and Pseudomonas exotoxin in Escherichia coli. Protein Expr Purif 2005, 39:189.

10. Kawakami K, Kawakami M, Joshi BH, Puri RK: Interleukin-13 receptortargeted cancer therapy in an immunodeficient animal model of human head and neck cancer. Cancer Res 2001, 61:6194-200.

11. Fichtner-Feigl S, Terabe M, Kitani A, Young CA, Fuss I, Geissler EK, Schlitt HJ, Berzofsky JA, Strober W: Restoration of tumor immunosurveillance via targeting of interleukin-13 receptor-alpha 2. Cancer Res 2008, 68:3467-75.

12. Terabe M, Matsui S, Park JM, Mamura M, Noben-Trauth N, Donaldson DD, Chen W, Wahl SM, Ledbetter S, Pratt B, Letterio JJ, Paul WE, Berzofsky JA: Transforming growth factor-beta production and myeloid cells are an effector mechanism through which CD1d-restricted T cells block cytotoxic T lymphocyte-mediated tumor immunosurveillance: abrogation prevents tumor recurrence. J Exp Med 2003, 198:1741-52.

13. Fichtner-Feigl S, Strober W, Kawakami K, Puri RK, Kitani A: IL-13 signaling through the IL-13alpha2 receptor is involved in induction of TGF-beta1 production and fibrosis. Nat Med 2006, 12:99-106.

14. MacDonald TT: Decoy receptor springs to life and eases fibrosis. Nat Med $2006,12: 13-4$

15. Zurawski SM, Vega F Jr, Huyghe B, Zurawski G: Receptors for interleukin-13 and interleukin- 4 are complex and share a novel component that functions in signal transduction. EMBO J 1993, 12:2663-2670.

16. Honjo Y, Bian Y, Kawakami K, Molinolo A, Longenecker G, Boppana R, Larsson J, Karlsson S, Gutkind JS, Puri RK, Kulkarni AB: TGF-beta receptor I conditional knockout mice develop spontaneous squamous cell carcinoma. Cell Cycle 2007, 6:1360-6.

17. Nakashima H, Terabe M, Berzofsky JA, Husain SR, Puri RK: A novel combination immunotherapy for cancer by IL-13Ra2-targeted DNA vaccine and immunotoxin in murine tumor models. J Immunol 2011, 187:4935-46.

18. Kawakami K, Taguchi J, Murata T, Puri RK: The interleukin-13 receptor alpha2 chain: an essential component for binding and internalization but not for interleukin-13-induced signal transduction through the STAT6 pathway. Blood 2001, 97:2673-9.

19. Kioi M, Kawakami K, Puri RK: Analysis of antitumor activity of an interleukin-13 (IL-13) receptor-targeted cytotoxin composed of IL-13 antagonist and Pseudomonas exotoxin. Clin Cancer Res 2004, 10:6231-8.

20. Nakashima H, Fujisawa T, Husain SR, Puri RK: Interleukin-13 receptor a2 DNA prime boost vaccine induces tumor immunity in murine tumor models. J Transl Med 2010, 8:116.

21. Puri RK, Leland P, Obiri NI, Husain SR, Kreitman RJ, Haas GP, Pastan I, Debinski W: Targeting of interleukin-13 receptor on human renal cell carcinoma cells by a recombinant chimeric protein composed of interleukin-13 and a truncated form of Pseudomonas exotoxin $A$ (PE38QQR). Blood 1996, 87:4333-9.

22. Husain SR, Joshi BH, Puri RK: Interleukin-13 receptor as a unique target for anti-glioblastoma therapy. Int J Cancer 2001, 92:168-175.
23. Kawakami K, Husain SR, Kawakami M, Puri RK: Improved anti-tumor activity and safety of interleukin-13 receptor targeted cytotoxin by systemic continuous administration in head and neck cancer xenograft model. Mol Med 2002, 8:487-94.

24. Kawakami K, Kawakami M, Husain SR, Puri RK: Potent antitumor activity of IL-13 cytotoxin in human pancreatic tumors engineered to express IL-13 receptor a2 chain in vivo. Gene Ther 2003, 10:1116-28.

25. Joshi BH, Kawakami K, Leland P, Puri RK: Heterogeneity in interleukin-13 receptor expression and subunit structure in squamous cell carcinoma of head and neck: differential sensitivity to chimeric fusion proteins comprised of interleukin-13 and a mutated form of Pseudomonas exotoxin. Clin Cancer Res 2002, 8:1948-56.

26. Pastan I, Hassan R, FitzGerald DJ, Kreitman RJ: Immunotoxin treatment of cancer. Annu Rev Med 2007, 58:221-37.

27. Husain SR, Puri RK: Interleukin-13 receptor-directed cytotoxin for malignant glioma therapy: from bench to bedside. J Neurooncol 2003, 65:37-48.

28. Fujisawa T, Joshi BH, Miyajima A, Puri RK: A novel role of interleukin-13 receptor a2 in pancreatic cancer invasion and metstasis. Cancer Res 2009, 69:8678-85.

29. Kioi M, Shimamura T, Nakashima H, Hirota M, Tohnai I, Husain SR, Puri RK: IL-13 cytotoxin has potent antitumor activity and synergizes with paclitaxel in a mouse model of oral squamous cell carcinoma. Int $\mathrm{J}$ Cancer 2009, 124:1440-8.

30. Fujisawa T, Nakashima H, Nakajima A, Joshi BH, Puri RK: Targeting IL-13Ra2 in human pancreatic ductal adenocarcinoma with combination therapy of IL-13-PE and gemcitabine. Int J Cancer 2011, 128:1221-31.

31. Sun ZJ, Zhang L, Hall B, Bian Y, Gutkind JS, Kulkarni AB: Chemopreventive and chemotherapeutic actions of mTOR inhibitor in genetically-defined head and neck squamous cell carcinoma mouse model. Clin Cancer Res 2012, 18:5304-13.

doi:10.1186/1479-5876-11-45

Cite this article as: Hall et al:: Targeting of interleukin-13 receptor a2 for treatment of head and neck squamous cell carcinoma induced by conditional deletion of TGF- $\beta$ and PTEN signaling. Journal of Translational Medicine 2013 11:45.

\section{Submit your next manuscript to BioMed Central and take full advantage of:}

- Convenient online submission

- Thorough peer review

- No space constraints or color figure charges

- Immediate publication on acceptance

- Inclusion in PubMed, CAS, Scopus and Google Scholar

- Research which is freely available for redistribution 